

\section{REVISTA \\ TEORÍA Y PRÁCTICA \\ DE LA \\ ARQUEOLOGÍA HISTÓRICA LATINOAMERICANA}

ISSN: 2250-866X (impreso) | ISSN: 2591-2801 (en línea)

AÑO VIII, VOLUMEN 9, PRIMAVERA DE 2019

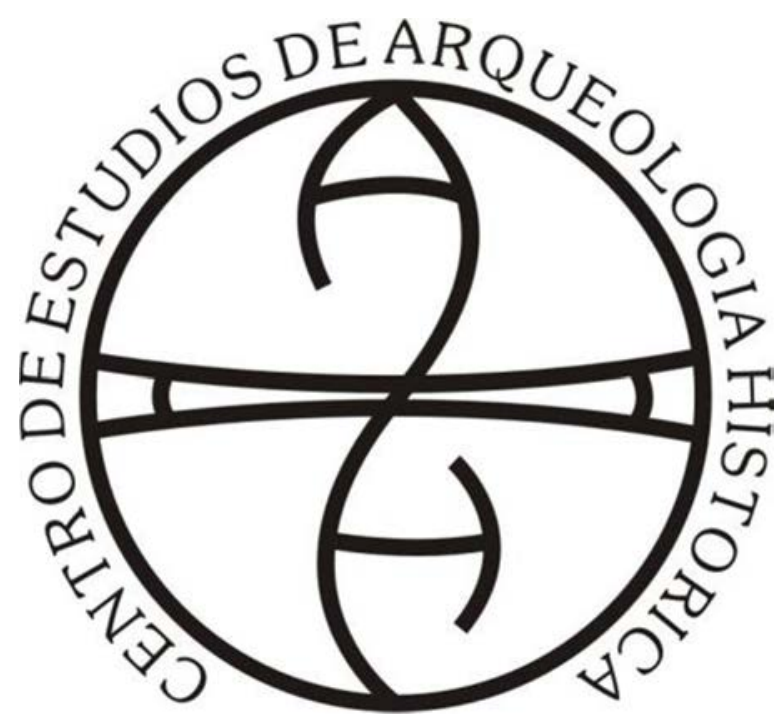

CENTRO DE Estudios de ARQueOlOGÍA HistóRICA

FACULTAD DE HUMANIDADES Y ARTES | UNIVERSIDAD NACIONAL DE ROSARIO 
(Universidad Nacional de Rosario, Universidad Nacional de Río Cuarto,

Universidad Nacional de San Juan, Universidad de la República, Universidad Nacional de Trujillo)

\author{
AUTORIDADES DE LA UNIVERSIDAD NACIONAL DE ROSARIO \\ RECTOR: Lic. Franco Bartolacci \\ VICE-RECTOR: Od. Darío Macía \\ SECRETARIO GENERAL: Prof. José Goity \\ SECRETARIA ACADÉMICO Y DE APRENDIZAJE: Dr. Marcelo Vedrovnik \\ SECRETARÍA DE CIENCIA TECNOLOGÍA E INNOVACIÓN \\ PARA EL DESARROLLO: Ing. Guillermo Montero.
}

\author{
AUTORIDADES DE LA FACULTAD DE HUMANIDADES Y ARTES \\ DECANO: Prof. Alejandro Vila \\ VICEDECANA: Prof. Marta Varela \\ SECRETARIA ACADÉMICA: Dra. Marcela Coria
}

\author{
AUTORIDADES DEL CENTRO DE ARQUEOLOGÍA HISTÓRICA \\ DIRECTORA: Dra. Ana Rocchietti \\ SECRETARIA: Prof. Nélida de Grandis \\ PROSECRETARIA: Lic. Marianela Bizcaldi
}

DIRECTORAS - EDITORAS:

Dra. Ana Rocchietti y Prof. Nélida De Grandis

SECRETARIA DE EDICIÓN: Dra. Irene Dosztal

Este número es co-edición de las ponencias

del VIII Congreso Nacional de Arqueología Histórica (2018) entre:

Centro de Estudios en Arqueología Histórica: Directora Ana Rocchietti

Centro de Estudios en Arqueología Regional: Director Fernando Oliva

Centro de Estudios en Arqueología Subacuática: Directora Mónica Valentini

Departamento de Arqueología, Escuela de Antropología: Director Fernando Oliva
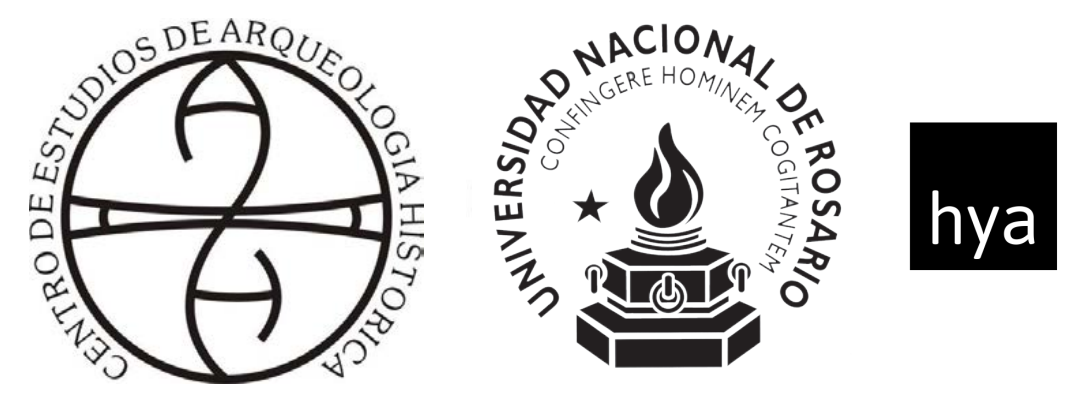

Facultad de Humanidades

y Artes_UNR
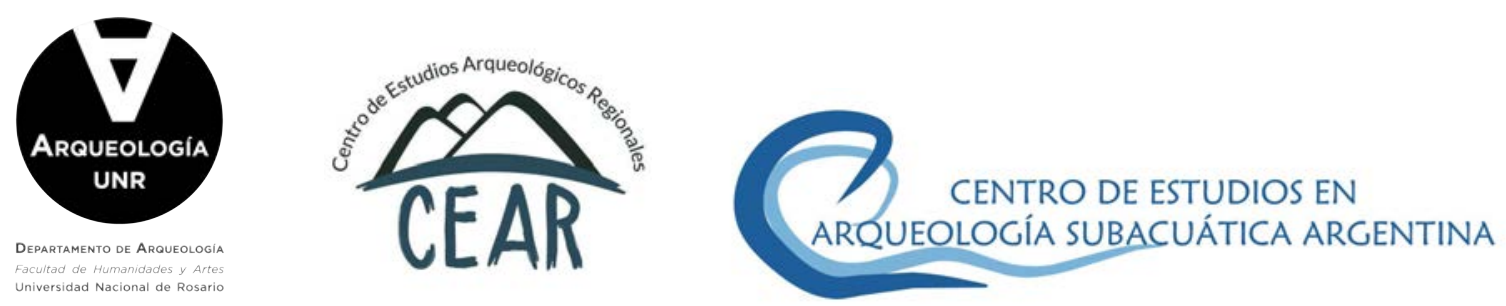
Comité Permanente de los Congresos Nacionales de Arqueología Histórica

Dr. Daniel Schávelzon (Universidad Nacional de

Buenos Aires)

Prof. María Teresa Carrara (Universidad Nacional de Rosario)

Prof. Carlos Baldassarre (Museo Municipal de Río

Grande, Tierra del Fuego) in memoriam

Dr. Mariano Ramos (Universidad Nacional de Luján, CONICET)

Dr. Horacio Chiavazza (Universidad Nacional de Cuyo)

Dra. Ana María Rocchietti (Universidad Nacional de

Rosario, Universidad Nacional de Río Cuarto)

Lic. Facundo Gómez Romero (Universidad Autónoma

de Barcelona)

\section{Comité Científico}

Dra. Tânia Andrade Lima (Universidade Federal do Rio de Janeiro)

Prof. Réginald Auger (CELAT/Département des

Sciences Historiques, Université Laval, Canadá)

Dr. Roberto Bárcena (Universidad Nacional de Cuyo, CONICET)

Dra. Marta Bonaudo (Universidad Nacional de Rosario, CONICET)

Dr. Leonel Cabrera (Universidad de la República, Uruguay)

Dr. Luis María Calvo (Universidad Católica de Santa

$\mathrm{Fe})$

Prof. Juan Castañeda Murga (Universidad Nacional de

Trujillo, Perú)

Dr. Carlos Ceruti (Museo de Ciencias Naturales y

Antropología "Prof. Antonio Serrano”. Paraná)

Dr. Horacio Chiavazza (Universidad Nacional de Cuyo)

Dra. Silvia Cornero (Universidad Nacional de Rosario)

Prof. Pedro Paulo Funari (Universidade Estadual de

Campinas, Brasil)

Lic. Jorge A. Gamboa Velásquez (Universidad Nacional

Santiago Antuñez de Mayolo, Perú)

Dr. Eduardo Alejandro García (Universidad Nacional de

San Juan, CONICET)

Prof. Nélida De Grandis (Universidad Nacional de

Rosario)

Dr. Juan Bautista Leoni (Universidad Nacional de

Rosario, CONICET)

Dra. Amancay Martínez (Universidad Nacional de San

Luis)

Dra. Catalina Teresa Michieli (Universidad Nacional de

San Juan, CONICET)

Lic. Fernando Oliva (Universidad Nacional de Rosario)
Ing. Adrián Pifferetti (Universidad Tecnológica Nacional Regional Rosario)

Dr. Mariano Ramos (Universidad Nacional de Luján, CONICET)

Dra. Ana María Rocchietti (Universidad Nacional de Rosario)

Dr. Daniel Schávelzon, (Universidad Nacional de Buenos Aires, CONICET)

Dra. Carlota Sempé (Universidad Nacional de La Plata)

Dr. Mario Silveira (Universidad Nacional de Buenos

Aires)

Dra. Silvia Simonassi (Universidad Nacional de Rosario)

Dra. Alicia Tapia (Universidad Nacional de Buenos Aires, Universidad Nacional de Luján)

Lic. Mónica P. Valentini (Universidad Nacional de Rosario)

Agrim. Benito Vicioso (Universidad Nacional de Rosario)

\section{Evaluaron este volumen}

Roberto Bárcena, María Teresa Boschin, Leonel Cabrera, Ulises Camino, María Rosa Carbonari, Carlos Ceruti, Horacio Chiavazza, Nicolás Ciarlo, Silvia Cornero, Eduardo Crivelli, Javier García Cano, Martín Gentinetta, María Laura Gili, Carlos Landa, Matilde Lanza, Melina Malandrino, Sebastián Pastor, Victoria Pedrotta, Josefina Piana, Mercedes Podestá, Mariano Ramos, Daniel Schavelzon, Diana Tamburini, Mónica Therrien, Mónica Valentini y María Teresa Boschin

\section{Diseño y diagramación}

Eugenia Reboiro

(eugenia.reboiro@gmail.com)

\section{Curadoría}

Ana Rocchietti e Irene Dosztal

Foto de tapa: Material arqueológico, del texto de Tapia et al.

\section{Propietario responsable:}

Facultad de Humanidades y Artes, Universidad Nacional de Rosario Centro de Estudios de Arqueología Histórica

Entre Ríos 758. Rosario, provincia de Santa

Fe (2000). Argentina.

Telf.: +54 (0341) 4802670

E-mail: ceahunr@gmail.com

Decreto Ley 6422/57 de Publicaciones

Periódicas 


\section{Índice}

Editorial

El origen del patrimonio histórico y su conservación integral en la contemporaneidad.

Yanina Aguilar

Prospección de basurales históricos de la ciudad de Rosario.

Soccorso Volpe y Gustavo Fernetti

Desconstrucción de un paisaje urbano. El Montevideo del extramuros colonial, aportes de la arqueología a su identidad actual. Ana Gamas

La Calota de Ameghino: reconsiderando un viejo tema desde la arqueología histórica.

Daniel Schávelzon

Análisis zooarqueológico de los restos hallados en una estructura sanitaria vinculada a momentos tempranos del actual barrio de Belgrano .71 Mario Silveira, Horacio Padula, Ricardo Orsini y Eva Bernat

La medida del tiempo: el reloj solar jesuita de La Cruz, provincia de Corrientes.

Fernando Oliva y María Cecilia Panizza

Sitio La Quinta: arqueología rural de campos serranos.

Flavio Ribero

Si no hay tabaco que no se note. Prácticas fumatorias en el fortin La Perra (1883-1885), La Pampa.

Alicia Tapia, Virginia Pineau y Melisa Ayelén Auge

El uso del tabaco y las pipas de caolin (clay pipes) en la frontera sur y oeste de la provincia de Buenos Aires en el siglo XIX.. .127

María del Carmen Langiano y Julio Fabián Merlo 
Patrimonio natural, urbano y arquitectónico de la costa en Mar del Plata. Federico Negroni

Huellas urbanas.

Ezequiel Serrot

Paisaje y patrimonio. La industria taninera en el siglo XX.

Provincia de Santa Fe.

Cristina Pasquali, Paola Milicic y Lara Ferré

Los desafíos de las arqueologías históricas latinoamericanas.

Andrés Zarankin

Sellos entre escombrales. Las lozas en el registro urbano

del Área Fundacional de Mendoza (siglo XIX y principios del siglo XX)

Lorena Puebla y Horacio Chiavazza

Evolución edilicia de la Plaza de Mayo de la Ciudad

de Buenos Aires (1580-1853).

Nicolás Ferrino

Gestión patrimonial en el rescate arqueológico y futuro Museo de Sitio

en Moreno 550, Ciudad Autónoma de Buenos Aires, Argentina.

María Eva Bernat, Ricardo Orsini, Horacio Padula y Mario Silvera 


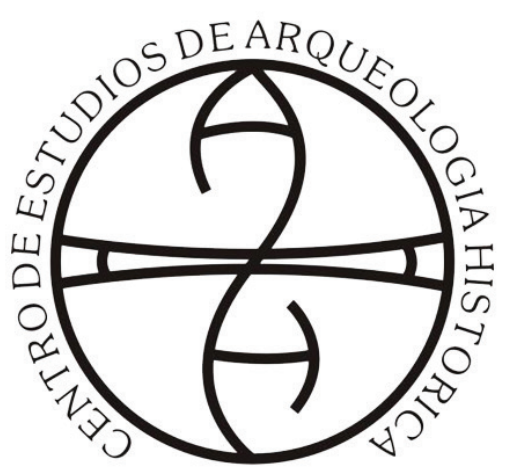

Centro de Estudios de Arqueología Histórica

Universidad Nacional de Rosario
Teoría y Práctica de la Arqueología Histórica

Latinoamericana | Año VIII. Vol. 9 | 2019

Revista del Centro de Estudios de Arqueología

Histórica, Facultad de Humanidades y Artes, Universidad Nacional de Rosario

https://teoriaypracticaah.unr.edu.ar/index.php/index ceahunr@gmail.com

ISSN en línea: 2591-2801

ISSN versión impresa: 2250-866X

\title{
EL ORIGEN DEL PATRIMONIO HISTÓRICO Y SU CONSERVACIÓN INTEGRAL EN LA CONTEMPORANEIDAD
}

\author{
Yanina Aguilar *
}

\section{Resumen}

Se ha ido reconceptualizando el estudio y la conservación del patrimonio histórico atravesando diversas coyunturas socio-históricas que lo sostenían solo desde su materialidad. Desde el concepto de herencia ancestral, de antigüedad, de monumento auténtico y original, de obras de arte de singular belleza, hacia una visión del patrimonio donde la característica de integralidad es hoy la clave para su conservación y preservación. En esta contemporaneidad el patrimonio integral es una herencia cultural donde se pone de manifiesto la creación colectiva y va marcando, a través de diversos imaginarios, las expresiones culturales que lo componen.

Los organismos internacionales dedicados al tratamiento normativo del patrimonio se han visto obligados a repensar en sus distintas cartas, conferencias y recomendaciones, la ampliación del concepto de patrimonio, incorporando gradualmente el contexto inmaterial que lo constituye y considerando las demandas de la sociedad en un tiempo dado y en un ámbito determinado.

Palabras clave: Patrimonio histórico, patrimonio integral, conservación integral, normativa internacional

\begin{abstract}
The study and preservation of the historical heritage has been reconceptualized crossing diverse sociohistorical junctures that supported it only from its materiality. From the concept of ancestral heritage, of antiquity, of an authentic and original monument, of works of art of singular beauty, to a vision of heritage where the characteristic of integrality is today the key to its conservation and preservation. In this contemporaneity, the integral heritage is a cultural heritage where the collective creation becomes evident and marks, through diverse imaginaries, the cultural expressions that compose it.
\end{abstract}

* Universidad Nacional de Río Cuarto. Facultad de Ciencias Humanas. Departamento de Historia. Laboratorio- Reserva de Arqueología.e-mail: feryanin@yahoo.com.ar 
Teoría y Práctica de la Arqueología Histórica Latinoamericana | Año VIII. Vol. 9 | 2019

ISSN en línea: 2591-2801 | ISSN versión impresa: 2250-866X

The international organizations dedicated to the normative treatment of heritage have been forced to rethink in their different letters, conferences and recommendations, the expansion of the concept of heritage, gradually incorporating the intangible context that constitutes it and considering the demands of society in a given time and in a certain area.

Keywords: historical heritage- integral heritage- integral conservation- international regulations

\section{Origen del patrimonio histórico}

Considerar en la actualidad una conservación integral del patrimonio, implica señalar que no siempre fue así, ya que el concepto mismo de patrimonio ha ido incorporando en su carácter ontológico diversos criterios sobre los bienes que lo componen y el tratamiento sobre los mismos.

La palabra patrimonio (del latín "patrimonium") se refiere al conjunto de bienes que una persona hereda de sus padres. Sanciona un sentido de la propiedad privada que es importante resaltar, porque la misma idea de propiedad no es universal, ni en el tiempo, ni en el espacio, sino que depende de factores culturales. Por otra parte alude a bienes materiales, que sin embargo, no son los únicos que se heredan, aunque sean los únicos que se codificaban en el derecho romano. Partiendo de este término se encuentran laras limitaciones, al tomar esta voz para referirse a aquellos bienes públicos o privados, pero de disfrute social, que unas generaciones van legando a otras, porque no basta con ampliar su atribución. (Ruiz Gil, 2005).

A principios del siglo XIX, el término hacía referencia a las antigüedades clásicas y progresivamente se fueron introduciendo conceptos arquitectónicos y artísticos referidos a la monumentalización.

Siguiendo a González-Varas (2000), el patrimonio histórico se desarrolló en el siglo XIX a partir de tres criterios que marcaron su recuperación, valorización y su conservación: a) Una interpretación ideológica o espiritualista que dotó a los monumentos del pasado de una fuerte carga emocional y simbólica, según la cual empezaron a ser considerados como manifestaciones gloriosas de la cultura nacional. b) Un progresivo interés turístico por conocer el patrimonio histórico de cada país, que se difundió gracias a la moda de los viajes pintorescos y a la publicación de numerosos libros, revistas y enciclopedias ilustrados, que presentaron a los monumentos artísticos como objetos de estudio literario, histórico e iconográfico. c) El desarrollo de la Historia del Arte como disciplina científica para el estudio de los monumentos y las obras de arte del pasado, tanto en sus aspectos estéticos como testimoniales, ideológicos, culturales, etc. (González Varas, 2000, p. 34-36).

¿Por qué se le atribuye al siglo XIX el origen del patrimonio histórico? El siglo XIX supone la consolidación del proyecto burgués, una estrategia contrarrevolucionaria de la burguesía que busca un nuevo consenso para cimentar el orden social, asustada tras 1815 por el avance de las ideas revolucionarias en las clases populares.

Se intenta reconstruir la historia de la nación, que gracias a la revolución liberal burguesa se constituye como unidad indivisible, basada en la raza, la lengua y la misma historia. Se busca en la historia el sentimiento de unidad nacional, asociado a valores como el sentimiento cristiano, el heroísmo, la libertad, el patriotismo y se encuentra generalmente en la edad media. Esos valores se encarnan en personajes históricos (en ese momento abundan las biografías), además cada uno de estos personajes históricos es la representación de lo colectivo. A través de desenterrar los acontecimientos más representativos en los que se plasman aquellos valores, se intenta configurar una memoria colectiva nacional. De ese modo el historicismo romántico sirve a los intereses del estado burgués y al mismo tiempo acomete la manipula- 
ción de la historia (Casado Galván, 2009) a partir del triunfo de la ideología de la burguesía liberal, de la clase emprendedora y con iniciativa creadora. La libertad propugnada está fuertemente vinculada al desarrollo del liberalismo económico, la apertura de nuevos mercados y el acceso a nuevos y abundantes espacios de materias primas. El colonialismo será su expresión y tiene su legitimación ideológica en el cientificismo positivista que sanciona la evolución y el progreso, y justifica la expansión de la civilización europea por todo el mundo. La progresiva conciencia de la capacidad de control del ser humano de los fenómenos que le rodean, le vuelven más consciente de su individualidad y de su libertad como sujeto para decidir (Hernando, 2004).

La más completa configuración de la concepción de la historia burguesa es la filosofía de la historia de Hegel, para el cual la historia sólo compete al hombre, pues sólo él es espiritual, así la separa de la naturaleza. La historia será el avance a través del tiempo hacia lo absoluto, que también incluye la libertad. El hombre, como ser racional, es responsable de sus actos -que son pensamiento en acción- y por tanto es responsable de su propia historia. Pero no individualmente, sino a través de la nación, o mejor, del Estado. La historia que Hegel considera como proceso dialéctico, recoge en cada momento una parte de aquel universal absoluto, universales o espíritus concretos, que tienen sus mismas cualidades y se reflejan en cada pueblo, en cada nación y se expresa en el espíritu nacional o Volksgeist, reflejo perfecto del espíritu de cada pueblo. Cada estado y cada pueblo tienen sus caracteres peculiares, cada uno expresa un momento en el desarrollo de la idea, ya que este desarrollo tiene lugar en el tiempo y en la existencia, y por ello en tanto historia, sus momentos y grados singulares son los espíritus de los pueblos.

El espíritu desplegado en la historia es lo que Hegel llama “espíritu del mundo” (Weltgeist) el cual es algo así como la providencia divina en cuanto dirige la historia. En cada momento de la historia hay un pueblo que, por ser dominante, expresa de manera adecuada al espíritu del mundo, el "espíritu de la época” (Zeitgeist).

De esta manera la historia universal es el proceso del desarrollo de la idea en el tiempo- de las grandes formas del Estado- en una marcha de creciente libertad.

Para Hegel la historia es una marcha progresiva y rigurosamente racional puesto que su curso está dominado y dirigido por la razón, por la idea. Desde este punto de vista, lo que en la historia cuenta, son los individuos en su aspecto racional o universal, y no en su individualidad, porque ésta no es más que el instrumento de la idea (Carpio, 2004, p. 311-312).

En ese marco historiográfico el patrimonio es tratado en el siglo XIX claramente como histórico material por un lado y natural por el otro. Su ontología se vio influenciada por el espíritu romántico que logro establecer una vinculación emocional entre las personas y su pasado histórico, como base del espíritu nacional de los pueblos. La vuelta al pasado se hizo entonces con el anhelo de reencontrar las raíces culturales y los elementos significativos que habían determinado a lo largo de la historia la forma de ser de las sociedades contemporáneas. Muchos filósofos e historiadores del siglo XIX coincidieron en afirmar que la identidad cultural de los pueblos se configuraba gracias a la concurrencia de una serie de expresiones colectivas, de carácter anónimo pero compartidas por todos, que sirven de base a un determinado folklore (Meinecke, 1943).

Los monumentos, además, constituyen objetos físicamente concretos que sirven para plasmar esa cultura común, a la vez que muestran la evolución de la misma a lo largo de los tiempos. Por eso en esta centuria tuvo lugar, sobre todo en los países de Europa, un proceso generalizado de identificación de cada civilización con sus monumentos más representativos. Por esta razón el Romanticismo apareció en muchas ocasiones cargado de una fuerte conciencia nacionalista, y las modas estéticas del ochocientos que se inspiraron en determinados modelos históricos, se consideraron legítimas herederas de los viejos esti- 
los nacionales para representar lo mejor de cada pueblo. En contra del clasicismo como estilo imperante en la Europa de aquella época, el Romanticismo empezó a creer en la relatividad de la historia, que podía traerse de nuevo al presente para mirarla desde una óptica diferente. El clasicismo fue desplazado como criterio estético dominante y el ideal del nuevo arte se inspiró en la imitación fidedigna del pasado medieval. De esta forma, en muchas zonas de Europa el absolutismo clasicista precedente fue sistemáticamente sustituido por el nuevo despotismo del gótico, que fue considerado el estilo nacional por antonomasia.

También la restauración monumental se impregnó de esta idea, haciéndose frecuente la reconstrucción "en estilo" de muchos edificios románicos y góticos, porque se consideraba que había una herencia espiritual compartida que daba continuidad a la creación original del artista medieval y la terminación de la misma por parte del arquitecto restaurador (Llull Peñalba, 2005).

Teniendo en cuenta lo mencionado, la idea de patrimonio ha ido evolucionando a lo largo de los siglos desde un planteamiento particularista, centrado en la propiedad privada y el disfrute individual, hacia una creciente difusión de los monumentos y las obras de arte como ejemplos de un patrimonio histórico, reflejo de la cultura nacional y símbolos de la identidad colectiva.

\section{La ampliación del patrimonio histórico en el Siglo XX}

Los términos de patrimonio histórico en el siglo XX empiezan a reconocer un aspecto fundamental en su análisis, porque más allá de apreciar la materialidad del pasado, se prioriza la idea de contextualización, de comprensión en conjunto, de su valor ante todo como conocimiento no solo de la existencia material, sino también la organización social, la cosmovisión, y la representación del mundo que fundan la identidad de un grupo social y lo diferencian de los demás. En este sentido también la aportación de los defensores del ecologismo cultural y los trabajos de campo con sociedades distintas a la tradición europea han contribuido a tener una visión más extensa de lo que implica la cultura, introduciendo la reflexión de la interacción hombre -naturaleza y de los espacios antrópicos como claros exponentes no sólo de la acción del hombre, sino sobre todo de su conocimiento y cosmovisión (García López, 2008).

Por lo tanto el concepto de patrimonio, su estudio y aplicación se ha ido reestructurando y adaptando de acuerdo a las coyunturas espacio- temporales mediante un complejo proceso de atribución de valores sometido al devenir de la historia, las modas y el propio dinamismo de las sociedades. Así, la selección del patrimonio al que se le otorga una serie de cualidades superiores, que justifica la necesidad de su conservación y transmisión para las generaciones futuras, puede cambiar con cierta frecuencia. A partir de este relativismo, la sociedad interacciona de manera distinta con el patrimonio, favoreciendo su protección, conservación en unos casos, y olvidando de su cuidado en otros.

La conservación del patrimonio ha estado centrada en el patrimonio material a partir de sus valores de antigüedad, originalidad, esteticidad, singularidad, belleza, excepcionalidad, entre otros. La tradición en este siglo ha situado el estudio y la acción sobre el patrimonio fundamentalmente en el objeto, olvidándose de la diversidad de actividades que sobre ellos se desarrollan y la pluralidad de agentes que pueden intervenir.

A partir de ello ¿cómo plantear el patrimonio integral?

En primer lugar en preciso señalar que el patrimonio cultural es considerado como el conjunto de bienes que representan simbólicamente una identidad. Los referentes simbólicos materiales o inmateriales se asocian a una serie de ideas y valores esenciales y por tanto inmutables, sacralizados. Así, el criterio fundamental no va a ser ni la antigüedad, ni la originalidad (aunque a veces ambas sean sobrevaloradas y confundidas como valor de autenticidad) lo fundamental es la eficacia simbólica, es decir la capacidad 
del símbolo para expresar de forma sintética y emocionalmente efectiva una relación entre ideas y valores (Prats, 1997).

En esta contemporaneidad es fundamental considerar al patrimonio histórico, más allá del objeto, definido de forma dinámica, como unidad de acción donde confluyen diferentes disciplinas académicas, colectivos profesionales, instituciones (públicas y privadas), asociaciones y organizaciones ciudadanas con el fin de perpetuar la memoria para el futuro.

La nueva terminología define un nuevo momento histórico y cultural donde es insuficiente la mera noción material y se potencia la concepción de producto cultural, de forma que se amplía el catálogo de bienes integrantes e incluso hasta lo producido hoy en día, siempre que suponga una plasmación de las vivencias y problemas de una sociedad en un contexto histórico.

En relación a lo planteado, es importante considerar cambios a nivel legislativo, a nivel de política de la cultura donde el tratamiento de lo patrimonial conlleve un tratamiento en su conjunto.

Para Benavides Solís (1995) la denominación del patrimonio histórico resulta, cuando menos, insuficiente, parcial y equívoca, porque sólo tiene en cuenta las sociedades históricas, olvidando otras que se sitúan en un espacio y tiempo diferentes, privilegiando las manifestaciones y los valores de tipo elitista y dejando en el olvido las más populares. En su opinión, sería preferible hablar de patrimonio integral porque este término abarca las distintas concepciones del tiempo y los diferentes niveles de expansión de los elementos artísticos, técnicos y etnológicos, hasta llegar a incluir el patrimonio natural transformado, que él identifica como el "medio ambiente entendido como simbiosis del patrimonio natural y cultural" (Benavides Solís, 1995, p. 33).

Plantear el patrimonio histórico desde una visión integral conlleva a considerar el criterio de conservación integrada donde el patrimonio cultural se presenta tangible e intangible al mismo tiempo, compuesto de valores ante una sociedad que lo selecciona y lo define por su carácter cultural, ambiental, espacial y temporal. Esta acepción permite la continuidad y la articulación temporal, al ser reconocido no solo como símbolo y testimonio del pasado, sino como documento que explica el presente y como factor que dinamiza la construcción de futuro.

Una perspectiva integral del patrimonio histórico que, expresado en el territorio, reconoce la importancia de su conservación integral.

\section{La conservación integral del patrimonio}

Organismos internacionales dedicados al tema, sobre todo la UNESCO y sus organismos consultores (ICOM, ICOMOS) han ido reflexionando sobre el evolución del concepto de patrimonio, no sólo por razones de deterioro por efecto del tiempo, la depredación y el medio ambiente adverso, sino por la cada vez más creciente participación de actores culturales y organismos nacionales e internacionales interesados en la preservación de los bienes culturales como transmisores de un legado histórico.

A continuación se pretende describir las acciones de los organismos internacionales anteriormente mencionados en cuanto a la protección y conservación del patrimonio integral, a través de la adopción de conferencias y recomendaciones internacionales, que han considerado a lo largo del tiempo, los valores de los bienes culturales, no solo en un plano tipológico y funcional, sino también identitario y socio-cultural.

El concepto de conservación integrada fue establecido en el año 1975 por el Consejo Internacional de Monumentos y Sitios (ICOMOS) a partir de la formulación de La Carta Europea del Patrimonio Arquitectónico. Si bien este marco regulatorio apunta a la conservación del patrimonio arquitectónico europeo, 
Teoría y Práctica de la Arqueología Histórica Latinoamericana | Año VIII. Vol. 9 | 2019

ISSN en línea: 2591-2801 | ISSN versión impresa: 2250-866X

cabe destacar que la incorporación del carácter de integralidad para la protección del patrimonio implicó un proceso de redefinición en las políticas patrimoniales en relación a su concepto y aplicación.

La carta adopta que el patrimonio no sólo está formado por monumentos más o menos relevantes, sino también por los conjuntos que constituyen ciudades y pueblos con sus tradicionales entornos naturales y construidos (Recuperado de: http://www.icomos.es/wp-content/uploads/2017/05/17.carta-europea-patrimonio.pdf).

Durante mucho tiempo sólo se han protegido y restaurado los monumentos más importantes, sin tener en cuenta su contexto. En consecuencia, éstos pueden perder gran parte de su carácter integral si este contexto es alterado. La conservación del patrimonio integral testimonia la presencia de la historia y de la memoria de los pueblos.

Por lo tanto el ejercicio de la conservación integral del patrimonio implica, en primera instancia, una capacidad reflexiva y analítica sobre la dimensión cultural del hecho urbano y/o arquitectónico; y en segunda instancia, la capacidad de entender la trascendencia de la intervención frente al compromiso de su protección, pero sobre todo, de su sustentabilidad en equilibrio y armonía con la sustentabilidad de la cultura, de la sociedad, del medio ambiente, de la ciudad y de la región, entre otras escalas.

La conservación integral del patrimonio comprende sus componentes culturales y naturales, pero no se trata de conservar por conservar; la conservación debe servir para transmitir y comunicar la enseñanza que nos brindan los elementos culturales del pasado. Esto implica que las acciones de fondo no se reducen al cómo se gesta la conservación técnica de bienes culturales, sino al cómo realizar su conservación como parte de un sistema mayor que engloba esas actividades, generando conciencia sobre el patrimonio integral bien sobre su diversidad de valores. Hemos pasado de la restauración monumental a la conservación integral del patrimonio (Tello Fernández, 2013, p. 103) y esto en relación a la evolución que ha ido manifestando el concepto mismo de patrimonio.

Desde esta perspectiva, el patrimonio integral hace referencia a lugares históricos urbanos y/o rurales-paisajes culturales y/o sectores urbanos (porciones de territorio o de la ciudad)- y conjuntos arquitectónicos y edificaciones, que por su representatividad histórica y socio-cultural, son reconocidos, declarados y/o protegidos como bienes de interés patrimonial. (García, 1992, p. 52).

La Conferencia Mundial sobre políticas culturales de la Comisión de Desarrollo de la Organización de las Naciones Unidas para la Educación, la Ciencia y la Cultura (UNESCO) en México, 1982 que mantiene su vigencia actual, aún en nuestros tiempos nos expresa que el patrimonio histórico no es sólo el conjunto de monumentos de singularidad excepcional, sino la totalidad dinámica y viva de la creación del hombre (Recuperado de https://unesdoc.unesco.org/ark:/48223/pf0000052505_spa).

En la misma línea, la Declaración de México en el año 1992, incluyó las obras de los artistas, arquitectos, músicos, escritores, así como las creaciones anónimas surgidas dela arte popular. Es decir, obras materiales e inmateriales que expresan la creatividad de un pueblo: la lengua, los ritos, las creencias, los lugares y monumentos históricos, la literatura, las obras de arte, los archivos y las bibliotecas (Tello, 2002, p. 6-7). Entonces, se considera que el patrimonio histórico está comprendido por toda aquella producción del hombre, entendida como creación artificial, ya sea material o inmaterial. Al respecto Pablo Macera (1999) considera que el patrimonio histórico no es sólo el patrimonio arquitectónico, ni siquiera el patrimonio visible, también incluye aquello que percibimos por otros sentidos.

En el año 1994, se realiza la Conferencia sobre Autenticidad de Nara, documento elaborado en Japón por el ICOMOS, señala en su acápite la importancia de la diversidad cultural que encarna la construcción del patrimonio integral considerando que la diversidad del patrimonio existe en el tiempo y en el espacio, y requiere respeto para las obras culturales y para todos los aspectos de sus sistemas de creencias. 
Teoría y Práctica de la Arqueología Histórica Latinoamericana | Año VIII. Vol. 9 | 2019

ISSN en línea: 2591-2801 | ISSN versión impresa: 2250-866X

Cuando los valores culturales parecen estar en conflicto, el respeto por la diversidad cultural exige el reconocimiento de la legitimidad de los valores culturales de todas las partes, definiendo las fuentes de información, todas las fuentes documentales escritas, orales y figurativas que hacen posible conocer la naturaleza, aspectos específicos que construyen el patrimonio integral a partir de su diversidad (Díaz de Cabeza y Rodríguez de Ortega, 2011, p.35).

Otro documento importante al tema en cuestión tiene que ver con la Carta de Brasilia del año 1995. Destacando que fue un documento regional a nivel latinoamericano, realizado en Brasil y en donde participaron países como Argentina, Chile, Paraguay y Uruguay. Un encuentro clave donde ese estableció los conceptos ejes para un enfoque patrimonial integrado desde nuestra región.

En esta Carta se señala que el patrimonio histórico, no solo se compone de edificios y sitios de envergadura material, a su vez son portadores de un mensaje o argumento cuya validez, en el marco de un contexto social y cultural determinado y que por aceptación por parte de la comunidad, lo convierte en patrimonio integral a partir de su significado inmaterial (Recuperado de http://www.icomoscr.org/doc/ teoria/VARIOS.1995.carta.brasilia.sobre.autenticidad.pdf).

Otro documento importante al momento de considerar la conservación integral del patrimonio es la carta de Burra. La misma fue adoptada el 19 de agosto de 1979 por el ICOMOS Australia en Burra. Fue actualizada en varias oportunidades, el 23 de febrero de 1981, el 23 de abril de 1988 y la última el 26 de noviembre de 1996.

Esta carta adquiere importancia sobre el tratamiento del patrimonio integral y su conservación en cuanto incorpora no solo la importancia sobre la significación que poseen los lugares, sitios, áreas, edificios y obras consideradas patrimonio material, sino también sitios naturales e indígenas. Los sitios de significación cultural enriquecen la vida de un pueblo, proveyendo un sentido de comunicación entre comunidad y paisaje, con el pasado y con experiencias vividas. Son reflejo de la historia material e inmaterial a la vez. Asimismo, hace referencia a la conservación del valor cultural del sitio con el adecuado mantenimiento en relación a las condiciones que presenten los mismos. El hecho de cuidar el patrimonio integral contribuye a reforzar la cohesión social.

Los propósitos de la carta en relación a los criterios de conservación y gestión, manifiestan un carácter integral en la conservación patrimonial. Esto se ve reflejado cuando la carta establece que la significación cultural de un sitio patrimonial se relaciona generalmente con aspectos intangibles, como cualidad simbólica indisociable del sentido de materialidad. Además de la significación natural de todo sitio, destacando la importancia de los ecosistemas, la diversidad biológica y la geodiversidad para la perpetuidad de sus valores tangibles e intangibles.

La conservación integral se sustenta a su vez, en el sentido de participación ya que la conservación, interpretación y gestión de un sitio, propugna la carta, debe contemplar la participación de la sociedad para la cual el sitio tiene especiales asociaciones y significados, o para aquellos que tienen responsabilidad social, espiritual o de otra naturaleza para con el sitio.

Las asociaciones significativas entre la sociedad y un sitio patrimonial deben ser respetadas, preservadas. También tener en cuenta el proceso de investigar e implementar las oportunidades de interpretación, conmemoración y celebración en los casos que sean requeridos (Recuperado de https://www.icomos.org/ charters/burra1999_spa.pdf).

Por ello considerar el patrimonio histórico como patrimonio integral, conlleva a implementar una conservación integral que, de acuerdo a Feilden y Jokiletho (2003) consiste en aquello que es físicamente parte de él y de su entorno. Un bien histórico, producto del pasado que ha sufrido cambios o deterioros ocasionados tanto por el desgaste natural como por su uso funcional. La suma de esos cambios se con- 
vierte de por sí en parte de su carácter histórico y de su material esencial y representa el valor intrínseco del bien; es el soporte de los testimonios históricos y de los valores culturales asociados, tanto del pasado como del presente.

La conservación integral, se inscribe en lo teórico, conceptual y metodológico en el ámbito de la política de la cultura, como política pública a implementar a múltiple escala y por ende, de intervención, en manifestaciones urbanas (tangibles e intangibles) y arquitectónicas, con el ánimo de generar procesos de cualificación, que contribuyan a un desarrollo sustentable del territorio. Por ello tiene su punto de partida en las aproximaciones ideológicas que una sociedad tiene sobre sí misma, en cómo estas se expresan, en las teorías que construye, en cómo estas teorías dan cuerpo a conceptualizaciones propias y contextualizadas de acuerdo a ratificaciones y normas internacionales como marco de políticas, normas y leyes, que enmarcan la toma de decisiones y las acciones de conservación.

De esta manera la conservación integral del patrimonio exige en la contemporaneidad la necesidad de una legislación para los espacios que ocupan los núcleos o conjuntos monumentales y de interés ambiental previendo, zonas de protección del paisaje urbano y/o rural a fin de procurar una integración de la misma con la naturaleza circundante.

Además son fundamentales las líneas de acción y la planificación como actividad de gestión que integra conservación e innovación.

Como contexto, es comprensible que una eficiente estrategia de gestión del patrimonio integral debe sostenerse en tres características: definición de políticas públicas y actualización de la legislación, construcción de capacidad institucional y organizacional incluyendo la descentralización administrativa y el trazado de políticas de la cultura en relación a las tareas de conservación del patrimonio integral (Hardoy y Dos Santos, 1983).

Es por ello que la conservación integrada se hace imprescindible en la gestión patrimonial a través de la recuperación de las áreas urbanas y/o rurales que en estado de degradación deben realizarse modificaciones no sustanciales de su composición histórica. La conservación integrada debe fundamentarse en sistemas de fondos públicos, que fomenten las iniciativas de las administraciones locales y debe promoverse también la participación de organizaciones privadas.

La conservación del patrimonio integral es considerada como uno de los objetivos principales de la planificación urbana y territorial (De Los Ríos, Poblet, Robinson, Valdivia, 2003). por lo tanto intervenir en el patrimonio integral, implicará, sobre todo, adecuarse a sus limitaciones e inconvenientes, que se pueda valorar su historicidad, su autenticidad y su supervivencia a través de la adecuada investigación, actuaciones de conservación y/o preservación material e inmaterial.

\section{Consideraciones finales}

Asistimos a una nueva visión del patrimonio en la que la comprensión del entorno abarca los aspectos geoecológicos, socioeconómicos y culturales, como un todo que no se puede separar. Entonces, los límites de lo que puede considerarse patrimonio histórico van más allá de su tipología y contenido material, en él la noción de integralidad abarca desde el ambiente hasta la inmaterialidad de su contexto y expresiones socio-culturales.

A su vez ha de ser el sistema jurídico quien tutele el patrimonio integral de los Estados en sus distintas escalas. En general las convenciones internacionales han identificado al patrimonio por sus elementos de excepcional y reconocido valor, sus ampliaciones en torno a la integralidad ha requerido de grandes debates en relación a procedimientos de identificación e interpretación de su conservación. Debates que 
siempre han considerado una visión eurocentrista a pesar de que hallan existidos instancias de análisis a nivel latinoamericano.

La conservación integral implica un análisis del territorio urbano y/o rural, que articulados con los demás sistemas y realidades urbanos y territoriales, como los de usos, movilidad, espacio público, equipamientos, áreas verdes cuerpos de agua, entre otros, permitan garantizar el desarrollo y la sustentabilidad social y urbana, y en ellas la sustentabilidad del patrimonio integral. De esta manera obliga a una comprensión e incorporación del patrimonio en el contexto del ordenamiento y la planeación territorial como objetivo de la autoridad de aplicación.

La expresión de inmaterialidad como uno de los componentes del patrimonio integral de una sociedad, su función social, y su capacidad de trasmitir significados y valores fundamentales como la identidad, la pertenencia, el arraigo y la memoria, su condición de capital socio-cultural que potencialmente puede dinamizar procesos de desarrollo, lo pone en la condición de recurso no renovable en su condición única, irrepetible e histórica.

La conservación integral y su puesta en valor, implica expresiones culturales tangibles e intangibles que representa en su integralidad valores esenciales de una sociedad, complejamente delimitados y definidos por su carácter social, ambiental, espacial y temporal. Trasmite valores y significados, que además de constituirse en dispositivos de la memoria, y fundamento de valores como la identidad, permite de manera prospectiva visualizar el futuro, constituyéndose en factor de desarrollo humano integral y sustentable.

\section{Referencias bibliográficas}

BENAVIDES SOLIS, J. (1995). Siete enunciados sobre la Teoría General del Patrimonio Cultural. Boletín Informativo del Instituto Andaluz del Patrimonio Histórico, (12), 32-37.

CARPIO, A, P. (2004). Principios de filosofia, una introducción a su problemática. Buenos Aires. Glauco. 2004, pp 311-312. Recuperado de: https://aeternaimperoblog.wordpress.com/2018/03/06/lafilosofia-de-la-historia-de-hegel/)

CASADO GALVÁN, I. (2009). Breve historia del concepto de patrimonio histórico: del monumento al territorio. En Contribuciones a las Ciencias Sociales. www.eumed.net/rev/cccss/06/icg.htm

DE LOS RÍOS, S., POBLET, R., ROBINSON, M., y VALDIVIA, F. (2003). Conservación integrada del patrimonio urbano de la nación: Caso de Lima. Lima Perú.

DÍAZ DE CABEZA, M.C., y RODRÍGUEZ DE ORTEGA, A. M. (2011). Raíces. Sobre los Valores del Patrimonio Cultural Iberoamericano. Córdoba. ADVOCATUS.

FEILDEN, B, M. y JOKILEHTO, J. (2003). Manual para el manejo de los sitios del Patrimonio Mundial cultural. Rome: ICCROM.

GARCÍA, F, D. (1992). Construir en lo Construido. Madrid: Editorial Nerea.

GARCÍA LÓPEZ, A. (2008). Patrimonio cultural: Diferentes perspectivas. Arqueoweb. Revista sobre Arqueología en Internet. 9(2). https://webs.ucm.es/info/arqueoweb/pdf/9-2/angelica.pdf

GONZÁLEZ VARAS, I. (2000). Conservación de bienes culturales. Teoría, historia, principios y normas. Madrid, Cátedra. 
HARDOY, J. E., y DOS SANTOS, M, R. (1983). Impacto de la urbanización en los centros históricos latinoamericanos. Proyecto Regional de Patrimonio Cultural y Desarrollo, PNUD-UNESCO. Northwestern University. Recuperado de: https://books.google.com.ar/books/about/Impacto_de_ la_urbanizaci\%C3\%B3n_en_los_centr.html?id=_7MkAQAAMAAJ\&redir_esc=y

HERNANDO, A. (2004). El Patrimonio entre la memoria y la identidad. https://es.scribd.com/ document/332680077/Hernando-2009-El-Patrimonio-Entre-La-Memoria-y-La-Identidad.

ICOMOS. (1996). La Carta de Burra. Recuperado de: https://www.icomos.org/charters/burra1999_spa. pdf.

ICOMOS. (1995). Carta de Brasilia. Recuperado de: http://www.icomoscr.org/doc/teoria/VARIOS.1995. carta.brasilia.sobre.autenticidad.pdf).

ICOMOS. (1975). Carta Europea del Patrimonio Arquitectónico. Recuperado de: http://www.icomos.es/ wp-content/uploads/2017/05/17.carta-europea-patrimonio.pdf.

LLULL PEÑALBA, J. (2005). Evolución del concepto y de la significación social del patrimonio cultural. Arte, Individuo y Sociedad, vol. (17) 175-204

MACERA, P. (1999). Estado y cultura en el Perú: Conservación del patrimonio cultural de la Nación. Presidencia del Congreso y la República, de la Comisión de Educación y Cultura. Lima. Perú.

MEINECKE, F. (1943). El Historicismo y su génesis. Madrid. Fondo de Cultura Económica.

Prats, Ll. (1997). Antropología y patrimonio. Barcelona: Editorial Ariel.

RUIZ GIL, J. L. (2005). Creer y crear. El patrimonio cultural en la encrucijada de la globalización. Ed Servicio de Publicaciones de la Universidad de Cádiz. Cádiz.

TELLO, S. (2002). En torno Patrimonio e Interdisciplinariedad. Escuela Profesional de turismo y hotelería de la Universidad de San Martín de Porres. Lima.

TELLO FERNÁNDEZ, M. I. (2013). El reto de una conservación patrimonial en el contexto del desarrollo territorial. Un ejercicio de prospectiva valorando la memoria. Cap\&Cua, ISSN-e 2145-5643 Vol. (8), No. 1, 2012, 23 págs. https://dialnet.unirioja.es/servlet/articulo?codigo=4762980

UNESCO. (1982). Conferencia Mundial sobre políticas culturales de la Comisión de Desarrollo de la Organización de las Naciones Unidas para la Educación, la Ciencia y la Cultura. México. Recuperado de: https://unesdoc.unesco.org/ark:/48223/pf0000052505 spa

Recibido: 10 de junio de 2019

Aceptado: 02 de septiembre de 2019 